\title{
Índice de Preço de Viagens e Turismo: metodologia, estimação e retrospecto
}

\author{
Travel and tourism price index: methodology, estimation and background \\ Índice de Precios de Viajes y Turismo: metodologia, estimación y retrospecto
}

\author{
Armênio de Souza Rangel ${ }^{1}$ \\ Heron Esvael do Carmo ${ }^{2}$
}

\begin{abstract}
Resumo
O objetivo desse artigo é apresentar a metodologia de construção do Índice de Preços de Viagens e Turismo e analisar os resultados de sua evolução no período de 1994 a 2009. O índice toma como base os preços coletados pelo IPCA do IBGE e a composição dos gastos realizados por viajantes e turistas. A importância desse tipo de informação é imediata na análise das principais tendências do turismo doméstico na medida em que os consumidores tomam suas decisões de gasto tendo por base o comportamento dos preços relativos. Deve-se ressaltar, também, a sua importância nas atividades de planejamento turístico de curto e longo prazos, principalmente por parte dos órgãos públicos.
\end{abstract}

Palavras-chave: índice de preço; viagens; turismo.

\begin{abstract}
The main purpose of this article is to present the methodology of construction of the Travel and Tourism Price Index and to analyze the evolution of this index in the period of 1994 to 2009. The index gets the prices collect by the IPCA of IBGE and the travelers and tourist expenditures composition. The importance of this type of information is clear in the analysis of the domestic tourism main tendencies because the consumers take their decisions of expenditure based in the relatives prices behavior. On the other hand, it is necessary to emphasize their importance in the long run and short run touristic planning activities mainly by the public organization.
\end{abstract}

Keywords: price index, travel, tourism.

\section{Resumen}

El objetivo de este trabajo es presentar la metodología de construcción del Índice de Precios de Viajes y Turismo y analizar los resultados de su evolución desde 1994 hasta 2009. El indice se basa en los precios recogidos por el IPCA del IBGE y la composición de los gastos realizados por viajeros y turistas. La importancia de este tipo de información es inmediato en el análisis de las principales tendencias en el turismo interno en la medida en que los consumidores toman sus decisiones de gasto en función del comportamiento de los precios

\footnotetext{
${ }^{1}$ Docente da Escola de Comunicações e Artes (ECA) da Universidade de São Paulo. Depto. de Relações Públicas, Propaganda e Turismo. E-mail: armênio@usp.br

2 Docente da Faculdade de Economia, Administração e Contabilidade (FEA) da Universidade de São Paulo. Depto de Economia. E-mail: hcecarmo@usp.br
} 
relativos. Cabe señalar, también, su importancia en las actividades de planificación del turismo a corto plazo y largo plazo, especialmente por los organismos públicos.

Palabras clave: índice de precios, viajes, turismo.

\section{Introdução}

Apesar de sua relevância, não há, no Brasil, qualquer tipo de acompanhamento sistemático da evolução dos preços de bens e serviços adquiridos por viajantes e turistas. A importância desse tipo de informação é imediata na análise das principais tendências do turismo doméstico, na medida em que os consumidores tomam suas decisões de gasto tendo por base o comportamento dos preços relativos. Da mesma forma, os fluxos de turismo emissivo e receptivo do Brasil também são fortemente influenciados em função de mudanças de preços relativos. Deve-se ressaltar, também, a sua importância nas atividades de planejamento turístico de curto e longo prazos, principalmente aquelas desenvolvidas por parte de órgãos públicos.

Os Índices de Preços de Viagens e Turismo existentes em outros países constituem, em geral, subconjuntos do Índice de Preços ao Consumidor, ou seja, os preços dos bens e serviços que compõem a cesta de consumo de viajantes e turistas são retirados do IPC. Nos EUA, o Travel Price Index (TPI), calculado pela Travel Industry Association of America's (TIA), é um subconjunto do Consumer Price Index (CPI), calculado pelo Bureau of Labor Statistics (BLS). Além desse índice, o Bureau of Economic Analysis (BEA), órgão responsável pelo cálculo das Contas Nacionais, estima os deflatores da indústria de viagens e turismo no cálculo das contas Satélites de Turismo ${ }^{3}$.

As categorias de bens e serviços consideradas no Travel Price Index são as seguintes: serviços recreativos, hospedagem (incluindo-se hotéis e motéis), alimentação e bebida fora do domicílio, transporte aéreo, transporte local, transporte intermunicipal e veículos automotivos. A estrutura de ponderação do TPI é determinada a partir de pesquisa de campo sobre a estrutura dos gastos de viajantes e turistas, conduzida pela TIA. Uma vez determinada a participação de cada categoria de gasto no gasto total de viajantes e turistas, torna-se possível

\footnotetext{
${ }^{3}$ O BLS iniciou, em dezembro de 2007, o cálculo de um índice de turismo de viajantes e turistas que visitam os EUA. No entanto, essa série foi descontinuada em 2008.
} 
obter o preço médio dos bens e serviços consumidos e, desta forma, analisar a mudança temporal dos preços relativos. A mesma metodologia pode ser utilizada com relação ao Brasil tomando-se o IPCA, calculado pelo IBGE, como base para a construção do Índice de Preços de Viagens e Turismo - IPVT.

O objetivo deste artigo é apresentar a metodologia de construção do Índice de Preços de Viagens e Turismo e analisar os resultados de sua evolução no período de 1994 a 2009. Este artigo é composto por três seções. Na primeira seção, é apresentada a metodologia de construção do IPVT. Na segunda, são apresentadas as estimativas obtidas e analisadas as tendências de evolução dos preços das principais categorias de gasto. Na terceira, é apresentada uma análise comparativa do comportamento do índice de viagens e turismo dos EUA e do Brasil.

\section{Metodologia e Fonte de Dados}

Para a construção de um índice de preços de viagens e turismo é necessário, de um lado, delimitar o conjunto de bens e serviços adquiridos por viajantes e turistas e os gastos realizados com relação a cada um desses bens e serviços. Desse modo, uma vez definida a composição do gasto de viajantes e turistas, torna-se necessário definir o método de cálculo da variação dos preços. A seguir, é apresentada a composição do gasto de viajantes e turistas e, logo após, a metodologia de cálculo adotada na construção do IPVT.

\subsection{Composição do gasto de viajantes e turistas}

Para se determinar a composição do gasto de viajantes e turistas, é necessária a realização de pesquisa de campo por meio de questionários selecionando-se uma amostra aleatória a partir de critérios estatísticos em que se fixa a margem de erro das estimativas. Diante da impossibilidade de realização de uma pesquisa primária de gastos de viajantes e turistas 4 , torna-se necessário lançar mão de fontes secundárias de informação. No Brasil, há duas fontes de informação que podem ser utilizadas na definição da estrutura de ponderação do IPVT. A primeira delas é a POF - Pesquisa de Orçamentos Familiares - realizada pelo IBGE para a determinação da estrutura de ponderação do IPCA. A última POF foi realizada nos anos 2002/2003 (IBGE,2005). Nessa pesquisa, são discriminados os gastos realizados pelos

\footnotetext{
${ }^{4}$ Devido ao elevado custo.
} 
consumidores em geral e, em particular, os gastos dos mesmos com viagens e turismo. A segunda fonte de informação é constituída pelas pesquisas FIPE/EMBRATUR realizadas nos anos de 1998, 2001 e 2006 (Rabay, Kadota et alii, 2006). Na última pesquisa, foram entrevistadas 37.000 famílias distribuídas pelas unidades da federação. A partir das informações coletadas obteve-se a seguinte estrutura dos gastos segundo a faixa de renda para não usuários de pacotes de viagens oferecidos por agências turísticas ${ }^{5}$ :

Tabela 1- Composição dos Gastos de Viajantes e Turistas por Classe de Renda. Em \%

\begin{tabular}{|l|r|r|r|r|r|}
\hline \multicolumn{1}{|c|}{ Itens de Gasto } & $\begin{array}{c}\text { De R } 350,00 \text { até } \\
1.400,00\end{array}$ & $\begin{array}{c}\text { De } \$ 1.400,01 \\
\text { até } \mathrm{R} \$ 5.250,00\end{array}$ & $\begin{array}{c}\text { De } \mathrm{R} \$ 5.250,01 \mathrm{a} \\
\mathrm{R} \$ 10.500,00\end{array}$ & $\begin{array}{c}\text { Acima de } \\
\mathrm{R} \$ 10.500,00\end{array}$ & Total \\
\hline Transportes & 42,27 & 33,13 & 28,40 & 25,21 & 31,29 \\
\hline Alimentação & 23,15 & 23,50 & 21,05 & 21,49 & 22,45 \\
\hline Hospedagem & 7,35 & 15,61 & 21,42 & 23,34 & 17,73 \\
\hline Compras & 12,37 & 13,88 & 13,84 & 14,23 & 13,79 \\
\hline Passeios e atrações & 4,93 & 5,50 & 5,96 & 5,97 & 5,64 \\
\hline Diversão noturna & 4,77 & 5,20 & 5,67 & 5,85 & 5,40 \\
\hline Transporte local & 2,39 & 1,54 & 2,21 & 2,64 & 2,05 \\
\hline Outros & 2,76 & 1,63 & 1,46 & 1,28 & 1,65 \\
\hline \multicolumn{1}{c|}{ Gasto Total } & 100,00 & 100,00 & 100,00 & 100,00 & 100,00 \\
\hline
\end{tabular}

Fonte: Pesquisa FIPE/EMBRATUR, 2006.

Como se pode observar, as despesas com transporte, alimentação e hospedagem absorvem, em média, $71,47 \%$ do gasto total de viajantes e turistas ${ }^{6}$. Esses são os principais itens que, devido à sua elevada participação no gasto total, devem determinar a maior parcela da variação de preços do IPVT.

Na pesquisa FIPE/EMBRATUR (2006), os gastos estão agrupados em categorias muito agregadas o que impossibilita, em algumas situações, identificar a mesma categoria de gasto nas estatísticas do IPCA. É o caso, por exemplo, dos gastos com compras que foram eliminadas do índice devido ao fato de que a pesquisa não discrimina os bens e serviços adquiridos por viajantes e turistas. Desse modo, eliminando-se, também, a categoria outros, na Tabela 2, encontram-se as ponderações dos itens a serem levados em consideração no IPVT:

\footnotetext{
${ }^{5}$ Apenas 3\% dos informantes utilizaram os serviços de agências de viagens

${ }^{6}$ Há uma pequena variância desses percentuais se considerarmos os diferentes estratos de renda.
} 
Tabela 2 - Estrutura de ponderação do IPVT

\begin{tabular}{|c|c|c|}
\hline Item de gasto & $\%$ inicial & $\%$ final \\
\hline Transporte & 31,29 & 37,00 \\
\hline Alimentação & 22,45 & 26,55 \\
\hline Hospedagem & 17,73 & 20,97 \\
\hline Passeios e atrações & 5,64 & 6,67 \\
\hline Diversão noturna & 5,4 & 6,39 \\
\hline Transporte local & 2,05 & 2,42 \\
\hline Total & 84,56 & 100,00 \\
\hline
\end{tabular}

Fonte: Pesquisa FIPE/EMBRATUR, 2006. Elaboração própria.

Por outro lado, há o interesse em se desagregar certas categorias de gasto para se obter uma medida mais apurada da mudança de preços relativos como é o caso, por exemplo, das despesas com transporte em que o transporte aéreo e rodoviário são bens substitutos próximos, de modo que a demanda por cada um desses meios de transporte é fortemente influenciada pela mudança de preços relativos entre os mesmos. Para desagregar os gastos com transporte, lançou-se mão das estatísticas da POF 2003-2004 do IBGE. Na

Tabela 3, encontram-se os dados sobre os gastos com transporte despendidos por viajantes e turistas.

Tabela 3 - Gastos com transporte de viajantes e turistas

\begin{tabular}{|l|c|c|}
\hline \multicolumn{1}{|c|}{ Gasto } & Valor & $\%$ \\
\hline Combustível de veiculo & $34.907,55$ & 33,02 \\
\hline Ônibus intermunicipal & $20.232,05$ & 19,14 \\
\hline Ônibus interestadual & $22.598,25$ & 21,38 \\
\hline Avião & $21.091,00$ & 19,95 \\
\hline Ônibus urbano & $3.780,85$ & 3,58 \\
\hline Taxi & $3.106,00$ & 2,94 \\
\hline TOTAL & $105.715,70$ & 100,00 \\
\hline
\end{tabular}

Fonte: POF-IBGE (2002/2003)

Essa desagregação do setor de transporte foi aplicada à participação dos gastos de transporte de $37,00 \%$, no gasto total da

Tabela 2. Desse modo, a estrutura de ponderação do IPVT encontra-se na Tabela 4. 
Tabela 4 - Estrutura de ponderação do IPVT

\begin{tabular}{|l|r|}
\hline \multicolumn{1}{|c|}{ Itens de Gasto } & $\%$ \\
\hline A. Transporte & 39,43 \\
\hline 1. Transporte rodoviário de passageiros & 15,97 \\
\hline 1.1. Transporte intermunicipal & 7,55 \\
\hline 1.2. Transporte interestadual & 8,43 \\
\hline 2. Transporte aéreo doméstico & 7,87 \\
\hline 3. Transporte local & 2,57 \\
\hline 4. Veículo próprio - combustível & 13,02 \\
\hline B. Alimentação & 26,55 \\
\hline C. Hospedagem & 20,97 \\
\hline D. Passeios e atrações & 6,67 \\
\hline E. Diversão noturna & 6,39 \\
\hline \multicolumn{1}{|c|}{ Total } & 100,00 \\
\hline
\end{tabular}

Fonte: Pesquisa FIPE/EMBRATUR, 2006; POF 2003-2004 IBGE. Elaboração própria.

Uma vez definidos os itens de gastos a serem considerados e a estrutura de ponderação, o próximo passo é identificar, na estrutura do IPCA, os bens e serviços similares ( 
Tabela 5). 
Tabela 5 - Identificação das categorias de gasto do IPVT no IPCA

\begin{tabular}{|l|c|c|c|}
\hline \multicolumn{1}{|c|}{ Categorias do IPVT } & Categorias do IPCA & Código IPCA & $\begin{array}{c}\text { Ponderação } \\
\text { IPVT }\end{array}$ \\
\hline A. Transportes & & & 39,43 \\
\hline 1. Transporte rodoviário de passageiros & & & 15,97 \\
\hline 1.1. Transporte intermunicipal & Ônibus intermunicipal & 5101006 & 7,55 \\
\hline 1.2. Transporte interestadual & Onibus interestadual & 5101007 & 8,43 \\
\hline 2. Transporte aéreo doméstico & Avião & 5101010 & 7,87 \\
\hline 3. Transporte local & & & 2,57 \\
\hline 3.1. Ônibus urbano & Ônibus urbano & 5101001 & 1,41 \\
\hline 3.2. Táxi & Táxi & 5101002 & 1,16 \\
\hline 4. Veículo próprio - combustível & Gasolina & 5104001 & 13,02 \\
\hline B. Alimentação & Alimentação fora do domicílio & 1201000 & 26,55 \\
\hline C. Hospedagem & Hotel & 7201090 & 20,97 \\
\hline 1. Hotel & Motel & 7.201 .068 & 46,03 \\
\hline 2. Motel & Excursão & 7201095 & 13,06 \\
\hline D. Recreação & Boate, danceteria e discoteca & 7201054 & 6,67 \\
\hline 1. Passeios e atrações & Total & 100,00 \\
\hline 2. Diversão noturna & & & \\
\hline
\end{tabular}

Fonte: IPCA-IBGE. Elaboração própria

Feitos

todos

esses

ajustes, 
Tabela 6, encontra-se detalhada a estrutura de ponderação do IPVT. A distribuição dos gastos com hospedagem, entre hotel e motel, foi feita tendo por base as ponderações do IPCA do IBGE. Por outro lado, as categorias passeios e atrações e diversão noturna foram agregadas na categoria recreação. 
Tabela 6 - Estrutura de Ponderação do IPVT

\begin{tabular}{|l|r|}
\hline \multicolumn{1}{|c|}{ Itens de Gasto } & \multicolumn{1}{|c|}{$\%$} \\
\hline A. Transportes & 39,43 \\
\hline 1. Transporte rodoviário de passageiros & 15,97 \\
\hline 1.1. Transporte intermunicipal & 7,55 \\
\hline 1.2. Transporte interestadual & 8,43 \\
\hline 2. Transporte aéreo doméstico & 7,87 \\
\hline 3. Transporte local & 2,57 \\
\hline 3.1. Ônibus urbano & 1,41 \\
\hline 3.2. Táxi & 1,16 \\
\hline 4. Veículo próprio - combustível & 13,02 \\
\hline B. Alimentação & 26,55 \\
\hline C. Hospedagem & 20,97 \\
\hline 1. Hotel & 16,03 \\
\hline 2. Motel & 4,93 \\
\hline D. Recreação & 13,06 \\
\hline 1. Passeios e atrações & 6,67 \\
\hline 2. Diversão noturna & 6,39 \\
\hline \multicolumn{1}{c}{ Total } & 100,00 \\
\hline
\end{tabular}

Fonte: Pesquisa FIPE/EMBRATUR, 2006; POF 2003-2004 IBGE. Elaboração própria.

\subsection{Método de cálculo}

Uma vez definida a composição do gasto em viagens e turismo, torna-se necessário definir a fórmula de agregação dos preços dos diferentes bens e serviços que compõem o IPVT. Para isso, adotar-se-á como referência a fórmula do Índice Laspeyres Modificado, que é uma fórmula factível de larga utilização no cálculo de índices de preços e que pode ser fundamentada nos três principais enfoques teóricos sobre números - índice, entre os quais o enfoque da teoria econômica dos números-índice, tal como apresentada por Konüs (1924).

Feitas essas considerações, os fundamentos da metodologia de cálculo do IPVT serão apresentados passo a passo a partir do caso de um consumidor individual, ou uma unidade de consumo, entre duas situações de tempo. Feito isto, serão abordados os problemas referentes 
ao cálculo de um indicador social que represente o conjunto dos consumidores de produtos e serviços de viagens e turismo e da elaboração de séries em cadeia do IPVT.

O ponto de partida, no enfoque da teoria econômica, para determinar um índice de preços para um subconjunto da cesta de produtos e serviços de consumo é a solução de um problema de minimização de custo que segundo Diewert (1993), pode ser decomposto em dois estágios: no primeiro, o consumidor visa minimizar o custo de atingir um determinado nível de utilidade e, no segundo estágio, escolhe o nível máximo de utilidade que é consistente com a parcela de seu orçamento referente ao subconjunto de mercadorias do IPVT. A solução do primeiro estágio permite definir uma função custo que depende do nível de utilidade e do preço. Esta função, representada a seguir.

$$
C\left(u_{t}, p_{t}\right) \equiv \min _{q}\left\{\sum_{i=1}^{n} p_{t}^{i} \times q^{i}: f(q)=u_{t} \equiv f\left(q_{t}\right)\right\}=\sum_{i=1}^{n} p_{t}^{i} \times q_{t}^{i}
$$

Tomando como referência a função custo (1) é possível definir um índice de preços para cada nível de utilidade $u_{t} \equiv f\left(q_{t}\right)$, segundo a proposta de Konüs (1924) em que $q_{t}$ e $p_{t}$ são, respectivamente, vetores de preços e quantidades referente ao período t, que, para simplificar, assume os valores 0 (período base de cálculo) e 1 (período de referência) no caso bissituacional. Na equação (1) o processo da otimização aplica-se a um consumidor (unidade de consumo). O índice de preços de Konüs é obtido procedendo-se a razão entre custos unitários obtidos a preços do período de referência e custos unitários obtidos no período base dado um nível de preferências (utilidade). Evidentemente o resultado o índice depende do nível de preferências considerado, no caso os do período base ou do período de referência.

$$
P_{K}\left(p_{0}, p_{1}, q_{t}\right) \equiv \frac{C\left(f\left(q_{t}\right), p_{1}\right)}{C\left(f\left(q_{t}\right), p_{0}\right)}
$$


Quanto o nível de utilidade tomado como referência é o do período base (0) é possível estabelecer uma correspondência entre o respectivo índice de Konüs e o índice de Laspeyres, mostrada a seguir.

$$
L_{0,1}=\frac{\sum_{i=1}^{n} p_{1}^{i} \times q_{0}^{i}}{\sum_{i=1}^{n} p_{0}^{i} \times q_{0}^{i}}
$$

Um resultado da teoria é que, do ponto de vista teórico, pode-se chegar a fórmulas exatas para determinadas especificações da função utilidade ou o seu dual, a função custo unitário.

Laspeyres é uma fórmula exata para funções de utilidade em que a utilização (consumo) de componentes do custo se dá em proporções fixas, conhecidas como funções de Leontief, que são funções lineares homogêneas e que resultam em preferências homotéticas. Assim, no caso de Laspeyres, como as quantidades dos produtos e serviços componentes da cesta do IVT são mantidas fixas, qualquer alteração de preços relativos, não leva a alteração nas quantidades demandadas de cada serviço ou produto, ou seja, a elasticidade preço é considerada zero.

A análise precedente se referiu a um caso de exata correspondência entre fórmulas de números-índice e uma especificação de função utilidade. Segundo os autores citados, a condição desta correspondência está associada à condição de homoteticidade das preferências dos consumidores. Em síntese, assumindo homoteticidade de preferências, um índice de Konüs pode ser determinado de modo mais simples como a razão entre os custos unitários "ótimos" dos períodos 0 e 1 e independe da cesta de bens e serviços de referência $q$ e do correspondente nível de utilidade.Ademais, homoteticidade de preferências do consumidor significa elasticidade renda unitária e caminhos de expansão partindo da origem e lineares.

O conceito ICV de Konüs para um consumidor individual pode ser utilizado como referência para a definição de índices para um grupo de consumidores (sociedade) nos moldes estabelecidos por Pollak (1989). Esse autor analisou dois conceitos de índice: plutocrático e democrático, que se diferenciam quanto ao peso implícito atribuído a cada consumidor relativamente ao conjunto de consumidores. Em índices plutocráticos, como é o caso do IVT, é atribuído a consumidor um peso equivalente a participação relativa de seus gastos de consumo nos gastos totais com viagens e turismo. 
Assim, se adotarmos a hipótese de que os preços são iguais para todas as classes de consumidores, que não é uma hipótese irrealista considerando que na maioria das transações os consumidores são tomadores de preços, a fórmula de Laspeyres aplicada ao cálculo do IVT pode ser apresentada como uma média aritmética ponderada de relativos de preços, em que os pesos correspondem à participação da despesa do conjunto de consumidores com cada produto/serviço de turismo no total das despesas de todos os consumidores com esses bens.

Na prática séries de números-índice de preços, como o IVT, são calculados por um processo de encadeamento de índices bissituacionais. A alternativa de comparação direta entre dois períodos distanciados no tempo, em que provavelmente ocorreram mudanças expressivas nas condições do problema de otimização do consumidor, não é considerada a mais adequada. A esse respeito, Keynes (1930, pág 109) escreveu: “The 'chain method' of compiling a series of index numbers, which was first introduced by Marshall, is an attempt to deal with the problem of changes in the character of consumption by assuming that the differences are small between any two consecutive positions in the series of positions to be compared".

A elaboração de séries encadeadas pode ser ancorada no conceito de subíndice definido por Pollak, desde que válida a hipótese de a função utilidade ser separável no tempo, ou alternativamente o conceito de "Índice Integral de Divisia" que supõe alterações instantâneas no nível de preços, cuja trajetória ao longo do tempo poderia ser aproximada, entre outras fórmulas, pela utilização da fórmula de Laspeyres encadeada. Para permitir a incorporação de novas especificações de produtos e serviços, na maioria das aplicações é utilizada a fórmula de Laspeyres-Modificado ${ }^{7}$, desenvolvida a partir da fórmula de Laspeyres, a partir do processo de geração de séries em cadeia do IVT, denominado I, para simplificar.

A série do IVT, encadeado desde o período zero até o período t, é gerada como segue:

$$
I V T_{0, t}=I_{0, t}=I_{0,1} \times I_{1,2} \times \ldots \times I_{t-2, t-1} \times I_{t-1, t}
$$

\footnotetext{
${ }^{7}$ Vide Endo \& Do Carmo (1984) e Diewert (1999).
} 


$$
I_{t-1, t}=I_{0, t} / I_{0, t-1}=\frac{\sum_{i=1}^{n} w_{0}^{i} \times r_{0, t}^{i}}{\sum_{i=1}^{n} w_{0}^{i} \times r_{0, t-1}^{i}}=\frac{\sum_{i=1}^{n} w_{0}^{i} \times r_{0, t-1}^{i} \times\left(r_{t-1, t}^{i}\right)}{I_{0, t-1}}
$$$$
I_{t-1, t}=\sum_{i=1}^{n} w_{t-1}^{i} \times r_{t-1, t}^{i}
$$

(6)

$$
w_{t-1}^{i}=w_{0}^{i} \times\left(\frac{r_{0, t-1}^{i}}{I_{0, t-1}}\right)
$$

$\mathrm{Na}$ fórmula de Laspeyres modificado a ponderação de cada subitem é modificada a cada período de acordo com a fórmula (7), em que:

$w_{0}^{i}$ é a ponderação (peso) base de cada mercadoria i, determinado no períodobase de ponderação;

$w_{t-1}^{i}$ é a ponderação (peso) ajustada para o mês-base de cálculo;

$r_{0, t-1}^{i}$ é o relativo acumulado de cada subitem i entre o mês base de ponderação e o mês base de cálculo (t-1)

$I_{0, t-1}$ As é o índice geral acumulado desde o mês base de ponderação até o mês base de cálculo (t-1)

\section{Evolução do Índice de Preços de Viagens e Turismo}

Definidos os bens e serviços, a estrutura de ponderação e o método de cálculo, torna-se possível calcular a evolução do IPVT ao longo do tempo. Foram estimadas séries retroativas ao mês de dezembro de 1994, em que se fixou a base de comparação do índice igual a 100, sendo que a base de ponderação encontra-se definida em dezembro de 2006. Os resultados dessa estimação encontram-se na Tabela 7. 


\section{O comportamento do IPVT, entre dezembro de 1994 e dezembro de} 2009, apresenta dois períodos bem diferenciados (

). O primeiro deles, que se estende até dezembro de 1999, o IPVT e O IPCA exibem comportamentos semelhantes (

). Posteriormente, a taxa média anual de variação do IPVT passa a superar a do IPCA e a diferença entre os dois índices é crescente. No período de dezembro de1998 a dezembro de 2009, o IPVT cresce a uma taxa média anual de $9,88 \%$ e o IPCA, $6,91 \%$.

Tabela 7 - Evolução do IPVT e do IPCA. 1994 - 2009

\begin{tabular}{|c|c|c|c|c|c|c|c|c|c|}
\hline Item & Transporte & $\begin{array}{c}\text { Alimentação } \\
\text { fora do } \\
\text { domićlio }\end{array}$ & Hospedagem & Recreação & IPVT & IPCA & $\begin{array}{c}\text { IPVT } \\
(1)\end{array}$ & $\begin{array}{c}\text { IPCA } \\
(1)\end{array}$ & IPVT/IPCA \\
\hline 1994 & 100,00 & 100,00 & 100,00 & 100,00 & 100,00 & 100,00 & & & 100,00 \\
\hline 1995 & 113,40 & 120,50 & 129,67 & 159,63 & 123,85 & 122,41 & 23,85 & 22,41 & 101,18 \\
\hline 1996 & 137,15 & 123,28 & 134,08 & 179,15 & 134,41 & 134,12 & 8,53 & 9,56 & 100,22 \\
\hline 1997 & 151,39 & 123,61 & 136,00 & 208,05 & 141,44 & 141,18 & 5,23 & 5,27 & 100,19 \\
\hline 1998 & 154,72 & 123,63 & 144,11 & 251,61 & 147,95 & 146,33 & 4,60 & 3,65 & 101,11 \\
\hline 1999 & 188,57 & 125,95 & 157,04 & 276,64 & 163,68 & 159,41 & 10,63 & 8,94 & 102,68 \\
\hline 2000 & 220,38 & 130,49 & 177,28 & 293,09 & 180,86 & 168,93 & 10,50 & 5,97 & 107,06 \\
\hline 2001 & 258,37 & 143,04 & 180,87 & 316,16 & 199,14 & 181,90 & 10,11 & 7,67 & 109,48 \\
\hline 2002 & 322,91 & 160,21 & 190,98 & 345,02 & 228,72 & 204,69 & 14,85 & 12,53 & 111,74 \\
\hline 2003 & 352,60 & 176,97 & 205,70 & 387,93 & 250,71 & 224,40 & 9,61 & 9,63 & 111,72 \\
\hline 2004 & 379,75 & 192,31 & 224,77 & 420,89 & 271,76 & 241,45 & 8,40 & 7,60 & 112,55 \\
\hline 2005 & 445,86 & 205,59 & 229,37 & 456,31 & 299,29 & 255,29 & 10,13 & 5,73 & 117,23 \\
\hline 2006 & 441,84 & 217,76 & 250,29 & 510,26 & 312,61 & 264,58 & 4,45 & 3,64 & 118,15 \\
\hline 2007 & 453,47 & 234,49 & 266,97 & 548,34 & 329,64 & 276,40 & 5,45 & 4,47 & 119,26 \\
\hline 2008 & 486,42 & 262,62 & 301,13 & 704,00 & 370,97 & 292,57 & 12,54 & 5,85 & 126,80 \\
\hline 2009 & 562,57 & 282,97 & 325,91 & 812,05 & 415,03 & 305,06 & 11,88 & 4,27 & 136,05 \\
\hline $1998-2009$ & 12,45 & 7,82 & 7,70 & 11,24 & 9,83 & 6,91 & & & \\
\hline
\end{tabular}

Fonte: Elaboração própria

(1) Taxa de variação anual; (2) Taxa média de variação anual 


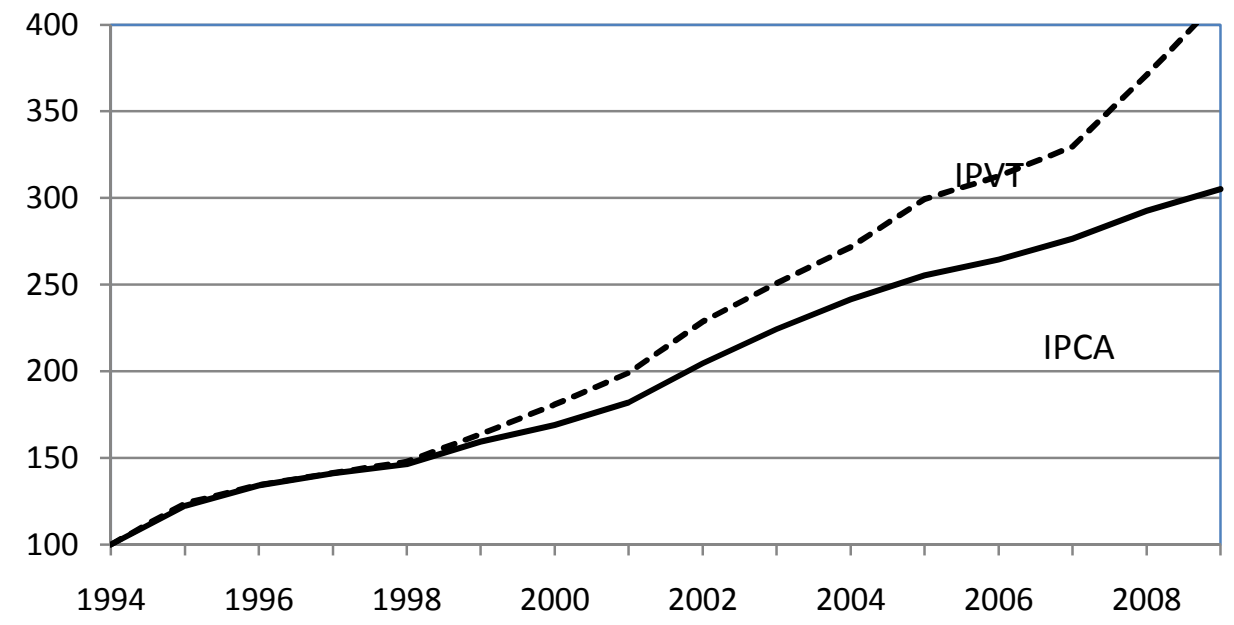

Gráfico 1 - Evolução do IPVT e do IPCA. 1994 a 2009

Deflacionando-se o IPVT pelo IPCA, observa-se, de forma mais nítida, o comportamento relativo desses dois índices. Até o ano de 1998, o IPVT se mantém estável em termos reais e, a partir do ano de 1999, o IPVT, cresce de forma bem mais acentuada do que o IPCA (Gráfico 2).

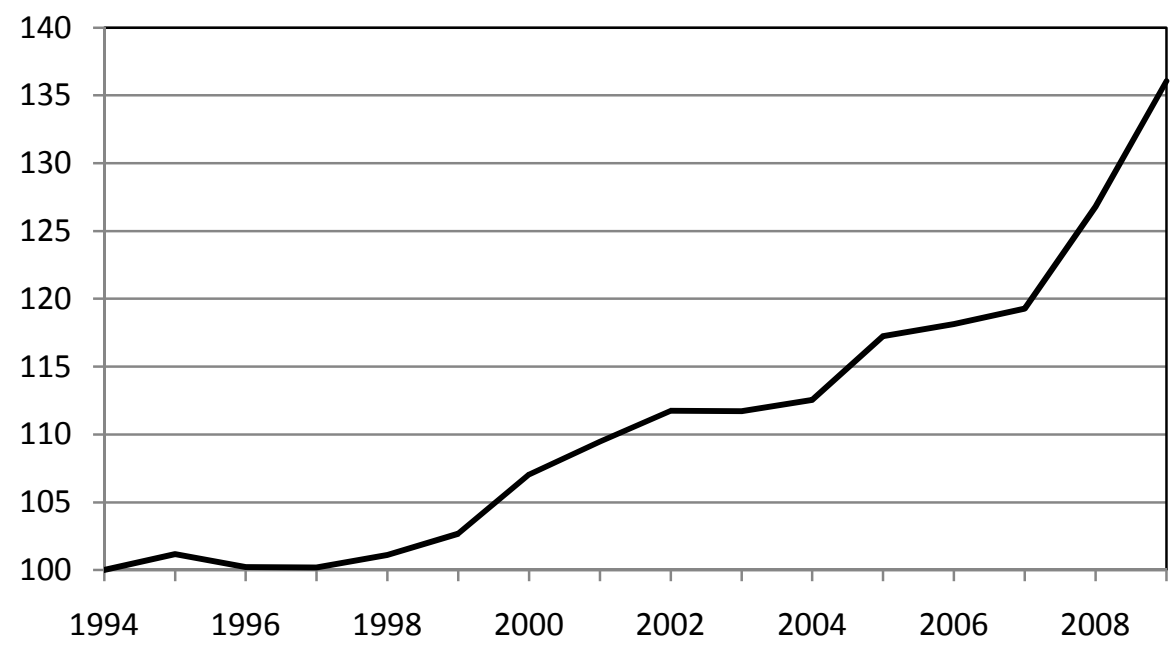

Gráfico 2 - Evolução do IPVT em termos reais 
Até o ano de 1998, o IPVT bem como o IPCA desaceleram o crescimento como decorrência do Programa de Estabilização de 1994. No entanto, a partir da desvalorização cambial de janeiro de 1999, ambos os índices crescem de forma acelerada até o ano de 2002. Nesse ano, como decorrência do clima de intranqüilidade que precedeu às eleições presidenciais desse ano, o IPCA elevou-se em $12,53 \%$ e o IPVT, $14,85 \%$ (Tabela 7). Posteriormente, ambos os índices reduzem a taxa anual de crescimento. Esse grande crescimento do IPVT, no período de 1998 a 2002, é decorrência imediata da desvalorização cambial de janeiro de 1999 que afetou de forma mais significativa o IPVT do que o IPCA. Na cesta de consumo considerada no IPVT, o item transporte tem grande peso. A desvalorização cambial não somente provocou um choque de elevação dos insumos importados em geral, mas especificamente em relação ao setor de transporte, o impacto foi significativo pois a Petrobrás repassou integralmente a desvalorização cambial aos preços do petróleo. Nos anos de 2002, 2003 e 2004, o IPVT parece se estabilizar em termos reais (Gráfico 2). No entanto, o atentado, em setembro de 2001, ao World Trade Center e, posteriormente, a Guerra do Iraque, iniciada em março de 2003, provocaram a explosão dos preços do petróleo afetando significativamente o comportamento do IPVT.

Após a desvalorização cambial, a taxa média anual de crescimento do IPVT de 1998 a 2009 foi de 9,83\% enquanto que o IPCA elevou-se em 6,91\%. Os itens alimentação e hospedagem seguiram de perto o IPCA, com taxas médias de variação anual de 7,82\% e 7,70\% respectivamente. Já o item transporte elevou-se a uma taxa média anual de $12,45 \%$ e o item recreação, $11,24 \%$ (Tabela 7$)^{8}$.

$\mathrm{Na}$

Tabela 8, o item transporte do IPVT encontra-se aberto segundo cada um de seus componentes. Os preços dos subitens transporte intermunicipal, interestadual, urbano experimentaram taxas de crescimento pouco acima do IPCA refletindo a escalada dos preços do petróleo e da desvalorização cambial de janeiro de 1999. No entanto, esses segmentos do setor de transporte se defrontam com controle de tarifas por parte do setor público de modo que a capacidade de repassar aumentos de custo aos preços é limitada. O mesmo não ocorre com a aviação civil que, a partir de 2002, acelera a elevação de preços conseguindo repassar aos preços a elevação dos preços do petróleo (Gráfico 3). De um lado, é um setor com um imenso poder de mercado e, de

\footnotetext{
${ }^{8} \mathrm{O}$ item passeios e atrações foi aproximado pelo dispêndio em excursão cujo preço também é afetado pela elevação do preço do petróleo.
} 
outro, a partir de 2002, a ANAC permitiu a livre fixação de tarifas. Desse modo, a taxa média de crescimento anual das tarifas aéreas atingiu $16,62 \%$.

Tabela 8 - Evolução do item transporte do IPVT

\begin{tabular}{|c|c|c|c|c|c|c|c|c|}
\hline Item & $\begin{array}{c}\text { Onibus } \\
\text { intermunicipal }\end{array}$ & $\begin{array}{c}\text { Ônibus } \\
\text { interestadual }\end{array}$ & $\begin{array}{c}\text { Ônibus a } \\
\text { longa } \\
\text { distância }\end{array}$ & Avião & $\begin{array}{c}\text { Ônibus } \\
\text { urbano }\end{array}$ & Táxi & $\begin{array}{c}\text { Veículo } \\
\text { próprio } \\
\text { (gasolina) }\end{array}$ & Transporte \\
\hline 1994 & - & - & 100,00 & 100,00 & 100,00 & 100,00 & 100,00 & 100,00 \\
\hline 1995 & - & - & 129,23 & 99,28 & 132,59 & 143,29 & 107,65 & 113,40 \\
\hline 1996 & - & - & 159,44 & 117,89 & 161,27 & 165,11 & 130,62 & 137,15 \\
\hline 1997 & - & - & 179,33 & 123,06 & 182,12 & 174,17 & 151,94 & 151,39 \\
\hline 1998 & 100,00 & 100,00 & 182,21 & 127,91 & 197,82 & 179,20 & 151,50 & 154,72 \\
\hline 1999 & 108,12 & 102,66 & 200,34 & 149,11 & 227,22 & 199,81 & 230,27 & 188,57 \\
\hline 2000 & 115,77 & 109,13 & 213,71 & 175,85 & 245,08 & 209,08 & 301,05 & 220,38 \\
\hline 2001 & 134,82 & 131,96 & 253,77 & 223,46 & 283,16 & 232,64 & 322,69 & 258,37 \\
\hline 2002 & 145,32 & 145,48 & 276,79 & 345,59 & 317,25 & 245,62 & 361,73 & 322,91 \\
\hline 2003 & 172,86 & 170,15 & 326,33 & 370,46 & 383,70 & 282,04 & 366,20 & 352,60 \\
\hline 2004 & 181,22 & 178,79 & 342,53 & 392,79 & 401,83 & 293,93 & 419,88 & 379,75 \\
\hline 2005 & 203,61 & 204,69 & 388,69 & 503,18 & 443,79 & 305,67 & 452,45 & 445,86 \\
\hline 2006 & 221,68 & 217,31 & 417,60 & 454,78 & 479,81 & 333,77 & 465,74 & 441,84 \\
\hline 2007 & 230,99 & 227,38 & 436,09 & 468,95 & 502,30 & 344,07 & 462,58 & 453,47 \\
\hline 2008 & 244,07 & 248,70 & 469,26 & 526,07 & 517,74 & 355,09 & 461,39 & 486,42 \\
\hline 2009 & 259,17 & 262,34 & 496,54 & 693,82 & 545,38 & 366,05 & 470,90 & 562,57 \\
\hline $1998-2009(1)$ & 9,04 & 9,16 & 9,54 & 16,62 & 9,66 & 6,71 & 10,86 & 12,45 \\
\hline
\end{tabular}

Fonte: Elaboração própria

(1) Taxa média de variação anual 


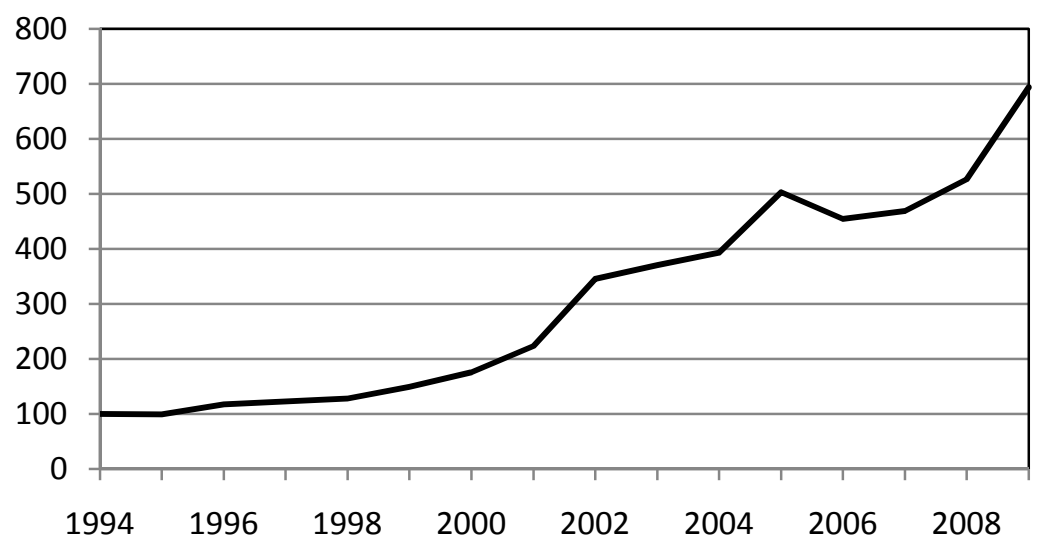

Gráfico 3 - Evolução do preço de passagens aéreas

O IPVT depende, basicamente, do comportamento dos seus itens mais relevantes. Evidentemente, quanto maior for o peso do item na composição do índice maior é a sua influência. A elasticidade do IPVT com relação a sada um de seus componentes $P_{i}$ é dada por

$$
\varepsilon=-\frac{\Delta I P V T}{\Delta P_{i}} \frac{P_{i}}{I P V T}
$$

sendo $w_{i}=\frac{\Delta I P Y T}{\Delta P_{i}}$ o peso do bem $i$ na composição do IPVT. Quano maior for o peso do componente maior deve ser, em princípio, a elasticidade. Quanto maior for a relação entre os preços do componente e o IPVT, maior é a elasticidade. Se $P_{i}=I P V T$, a elasticidade é exatamente igual ao peso do componente considerado no índice. Na Tabela 9, encontram-se estimadas as elasticidades do IPVT com relação a cada um de seus componentes ${ }^{9}$.

Tabela 9 - Elasticidades do IPVT com relação a seus componentes

\begin{tabular}{|c|c|c|c|}
\hline \multirow{2}{*}{ Componentes do IPVT } & \multicolumn{2}{|c|}{ Pesos } & \multirow{2}{*}{ Elasticidade } \\
\cline { 2 - 3 } & Dez 2006 & Dez 2009 & \\
\hline Transportes & 0,394 & 0,376 & 0,325 \\
\hline Alimentação & 0,265 & 0,259 & 0,391 \\
\hline Hospedagem & 0,210 & 0,209 & 0,194 \\
\hline Recreação & 0,131 & 0,156 & 0,090 \\
\hline Total & 1,000 & 1,000 & 1,000 \\
\hline
\end{tabular}

Fonte: Elaboração própria

\footnotetext{
${ }^{9}$ As elasticidades foram estimadas por MQS tomando-se o logaritmo das variáveis.
} 
Como se pode observar, os itens transporte e alimentação com pesos no índice de 0,376 e 0,259 apresentam as maiores elasticidades, 0,325 e 0,391 respectivamente. Esses são os dois itens que determinam, basicamente, o comportamento do IPVT.

Todo índice de preços deve utilizar, na agregação dos diferentes preços, uma função homogênea do $1^{\circ}$ grau, ou seja, a função deve ser tal que se os preços dos diversos componentes do índice se elevam, o índice deve se elevar na mesma proporção. Essa propriedade é garantida pela fórmula do Índice de Laspeyres em que a soma dos pesos dos diversos itens e das elasticidades do índice com relação a cada um de seus componentes são iguais à unidade (Tabela 9).

\section{Comparação do IPVT com o TPI}

Nesta seção, é feita uma comparação entre o comportamento do IPVT e TPI para o período de 1998 a 2009. No período de 1998 a 2009, o IPVT eleva-se de forma bem mais significativa do que o TPI. A taxa média de variação anual do IPVT foi de $9,83 \%$ enquanto que a do TPI foi de 2,97\% (Tabela 10). Como se pode observar, os preços dos bens e serviços de viagens e turismo elevam-se de forma bem mais significativa no Brasil do que nos EUA (Gráfico 4). Como já foi observado, até o ano de 2002, há uma disparada do processo inflacionário no Brasil como decorrência da desvalorização cambial de janeiro 1999, da escalada do preço do petróleo e das eleições presidenciais de 2002. Após o ano de 2002, a inflação no Brasil reduz o seu ritmo de crescimento, mas, mesmo assim, situa-se bem acima da taxa de inflação observada nos EUA.

Tabela 10 - Evolução do IPVT e do TPI. 1998 a 2009

\begin{tabular}{|c|c|c|c|c|c|c|c|}
\hline Ano & IPVT & TPI & IPCA & CPI & IPVT/TPI & $\begin{array}{c}\text { Taxa de } \\
\text { câmbio } \\
\text { nominal }\end{array}$ & $\begin{array}{c}\text { Taxa de } \\
\text { câmbio real }\end{array}$ \\
\hline 1998 & 100,00 & 100,00 & 100,00 & 100,00 & 100,00 & 1,16 & 100,00 \\
\hline 1999 & 110,63 & 104,00 & 108,94 & 102,19 & 106,38 & 1,81 & 147,00 \\
\hline 2000 & 122,25 & 114,01 & 115,45 & 105,64 & 107,23 & 1,83 & 147,08 \\
\hline 2001 & 134,60 & 115,43 & 124,31 & 108,62 & 116,61 & 2,35 & 173,68 \\
\hline 2002 & 154,60 & 115,14 & 139,88 & 110,35 & 134,27 & 2,92 & 187,47 \\
\hline 2003 & 169,46 & 117,14 & 153,35 & 112,85 & 144,66 & 3,08 & 183,36 \\
\hline
\end{tabular}




\begin{tabular}{|c|c|c|c|c|c|c|c|}
\hline 2004 & 183,69 & 120,64 & 165,01 & 115,87 & 152,26 & 2,93 & 165,59 \\
\hline 2005 & 202,30 & 127,20 & 174,47 & 119,80 & 159,04 & 2,44 & 131,94 \\
\hline 2006 & 211,30 & 133,43 & 180,81 & 123,67 & 158,36 & 2,18 & 118,41 \\
\hline 2007 & 222,81 & 139,43 & 188,89 & 127,20 & 159,81 & 1,95 & 105,03 \\
\hline 2008 & 250,75 & 147,26 & 199,94 & 132,08 & 170,28 & 1,83 & 92,84 \\
\hline 2009 & 280,53 & 138,00 & 208,48 & 131,61 & 203,28 & 2,00 & 84,68 \\
\hline \%aа (1) & 9,83 & 2,97 & 6,91 & 2,53 & 6,66 & 5,06 & $-1,50$ \\
\hline
\end{tabular}

Fonte: IPCA-IBGE; Câmbio-BCB; TPI-USTA; CPI-BLS. Elaboração própria

(1) Taxa média de variação anual

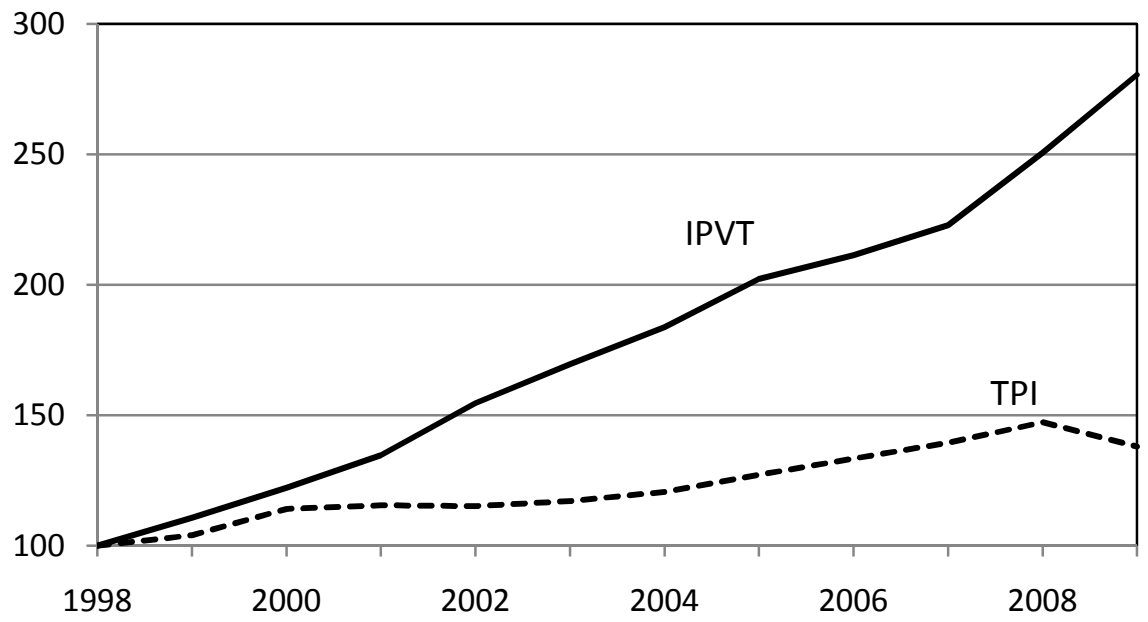

Gráfico 4 - Evolução do IPVT e do TPI. 1998-2009

A evolução do IPVT relativamente ao TPI pode ser melhor analisada deflacionando-se o IPVT pelo TPI (Gráfico 5). Até o ano de 2000, o IPVT cresce pouco acima do TPI. Posteriormente, o IPVT cresce de forma acentuada acima do TPI até o ano de 2005. Nos anos de 2005, 2006 e 2007 a relação fica estável crescendo posteriormente. 


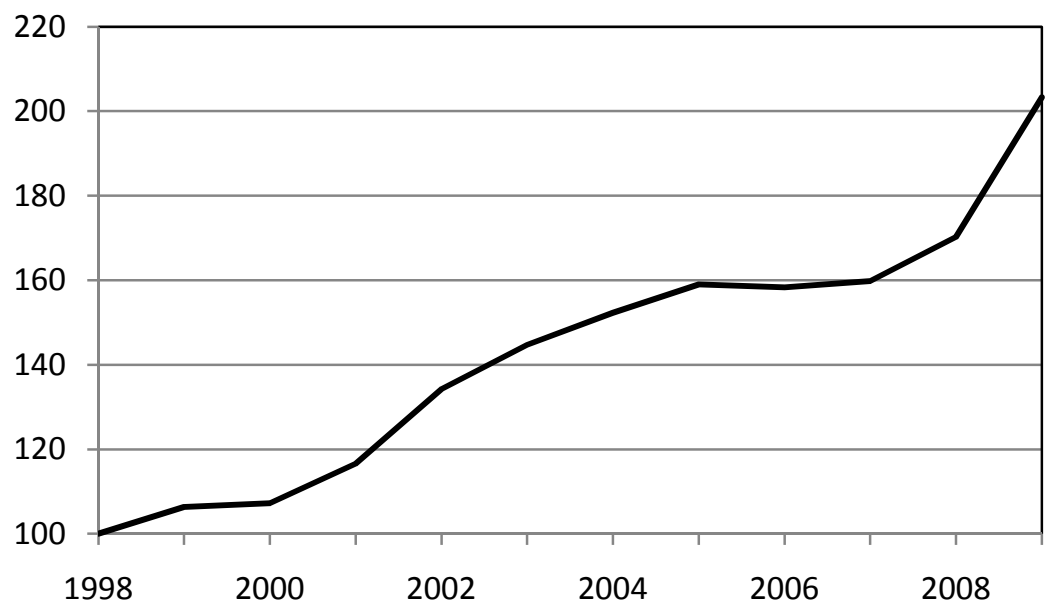

Gráfico 5 - Evolução do IPVT deflacionado pelo TPI

Dado que os preços crescem de forma bem mais acentuada no Brasil do que nos EUA, ao que tudo indica, esse resultado deveria desestimular o turismo receptivo e estimular o turismo emissivo do Brasil com relação aos EUA. No entanto, essa comparação não é válida, pois devemos levar em consideração a taxa de câmbio praticada entre os dois países. Até o ano de 2002, há uma grande desvalorização da taxa de câmbio nominal no Brasil e uma elevação mais acentuada dos preços domésticos de bens e serviços turísticos do que nos EUA. A elevação mais acentuada dos preços no Brasil tenderia a valorizar a taxa de câmbio real. No entanto, a forte desvalorização da taxa de câmbio nominal conduziu a uma acentuada desvalorização da taxa de câmbio real (Gráfico 6). Portanto, até o ano de 2002, há um forte estímulo para o turismo receptivo do Brasil com relação aos EUA. Posteriormente, seja em função do crescimento mais acelerado dos preços domésticos, seja pela forte valorização da taxa de câmbio nominal, observa-se uma forte valorização da taxa de câmbio real estimulando o turismo emissivo do Brasil. 


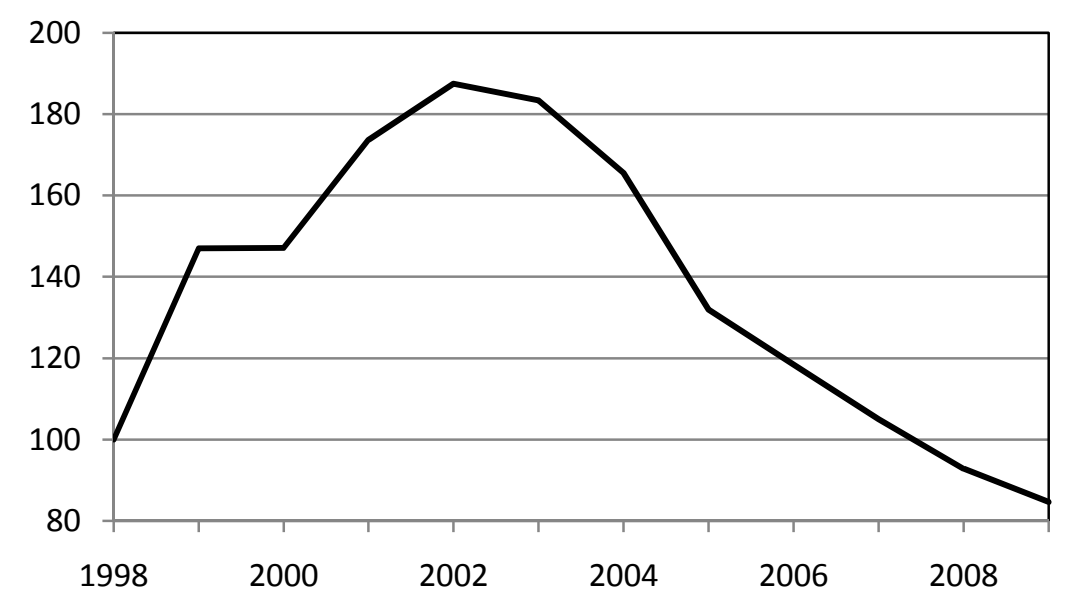

Gráfico 6 - Evolução da taxa de câmbio real de viagens e turismo

A relação entre movimentos da taxa de câmbio real e movimento de viajantes e turistas pode ser observada pela entrada de turistas provenientes dos EUA para o Brasil no período de 1998 a 2009. Até o ano de 2005, eleva-se a entrada de viajantes e turistas norte-americanos. Posteriormente, diante da forte valorização da taxa de câmbio real, observa-se uma redução na entrada de viajantes e turistas. Evidentemente, a entrada de viajantes e turistas não depende somente da taxa de câmbio real mas, também, de outras variáveis como renda, custo de destinos alternativos etc.

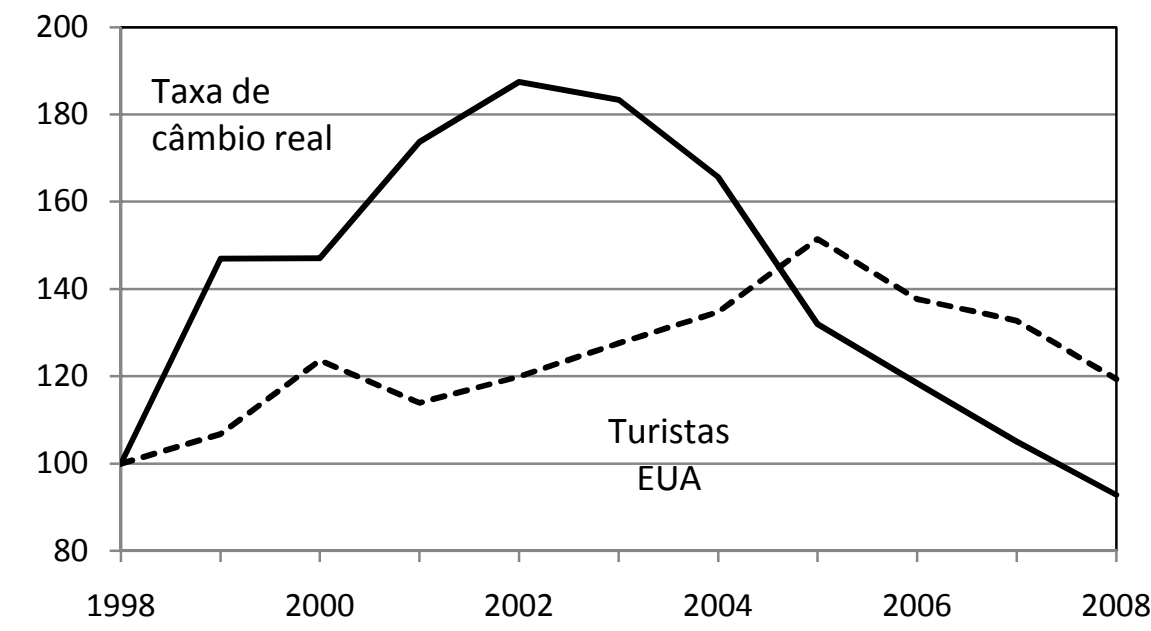

Gráfico 7 - Evolução da taxa de câmbio real e do emissivo dos EUA para o Brasil. 1998 a 2008 
Finalmente, cabe uma observação sobre os modelos econômicos usualmente utilizados na previsão do movimento de viajantes e turistas. Devido à inexistência de índices de preços sobre viagens e turismo para a grande maioria dos países ${ }^{10}$, normalmente utiliza-se como proxy os índices de preço ao consumidor. No

Gráfico 8, pode-se observar a evolução da relação entre os índices preços de viagens e turismo nos EUA e Brasil bem como entre os respectivos índices de preços ao consumidor. Ao se utilizar a relação IPCA/CPI no lugar da relação IPVT/TPI comete-se, desde logo, um erro de aproximação. É bem verdade que a correlação entre ambas as séries de 0,969 é elevada, o que parece se constituir numa boa aproximação que valida os inúmeros modelos de demanda estimados ${ }^{11}$.

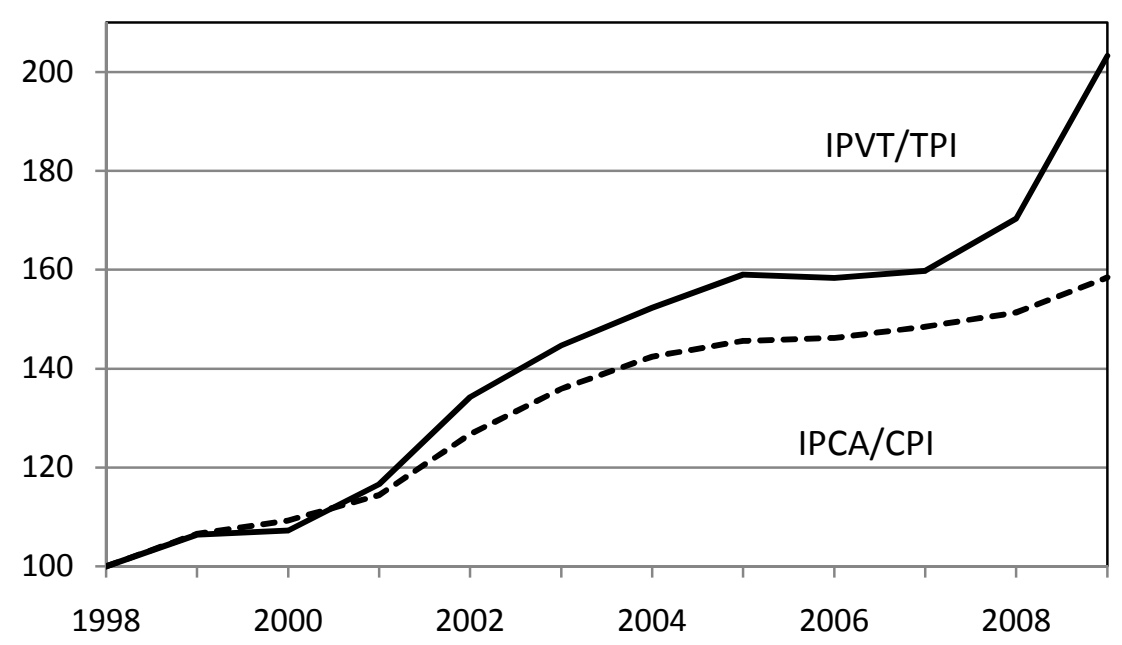

Gráfico 8 - Evolução relativa dos índices de preço ao consumidor e dos índices de preços de viagens e turismo. Brasil e EUA. 1998 a 2009

\section{Conclusões}

Tendo por base a estrutura do gasto de viajantes e turistas, dada pela Pesquisa FIPE/EMBRATUR (2006), e os preços coletados pelo IPCA do IBGE, foi possível estruturar

\footnotetext{
${ }^{10}$ Além dos EUA, pode-se citar a Espanha, Macao, Canadá, Virgin Islands etc.

${ }^{11}$ Entre outros, vide CROUCH (1992), ONG (1995), SONG \& LIB (2008) e LIM (1997).
} 
um índice de preços de viagens e turismo IPVT e acompanhar a sua evolução no período de dezembro de 1994 a dezembro de 2009. Na composição do índice, os pesos iniciais foram fixados em dezembro de 2006 e a base de comparação igual a 100 fixada em dezembro de 1994. O índice é composto de quatro categorias de gasto subdivididas, por sua vez, em vários itens: transporte $(39,43 \%)$, alimentação $(26,55 \%)$, hospedagem $(20,97 \%)$ e recreação $(13,06 \%)$. Foi adotada a fórmula do Índice de Preços Laspeyres Modificado em que os pesos de cada componente do índice variam em função da mudança de preços relativos.

O comportamento do IPVT revela dois períodos diferenciados antes e após a desvalorização cambial de janeiro de 1999. Até dezembro de 1998, o IPVT e o IPCA exibem comportamentos semelhantes. Posteriormente, o IPVT cresce de forma mais acelerada do que o IPCA. No período de dezembro de1998 a dezembro de 2009, o IPVT cresce a uma taxa média anual de $9,83 \%$ e o IPCA, 6,91\%. A desvalorização cambial de janeiro de 1999 afetou de forma mais significativa o IPVT do que o IPCA em função do grande peso das despesas com transporte na composição do IPVT. O atentado, em setembro de 2001, ao World Trade Center e, posteriormente, a Guerra do Iraque, iniciada em março de 2003, provocaram a explosão dos preços do petróleo afetando significativamente o comportamento do IPVT. Os itens alimentação e hospedagem seguiram de perto o IPCA, com taxas médias de variação anual de 7,82\% e 7,70\% respectivamente. Já o item transporte elevou-se a uma taxa média anual de $12,45 \%$ e o item recreação, $11,24 \%$.

Foram estimadas as elasticidades do IPVT com relação a seus componentes básicos encontrando-se os seguintes valores: alimentação $(0,391)$, transporte $(0,325)$, hospedagem $(0,194)$ e recreação $(0,09)$. Como se pode observar, o IPVT é extremamente sensível às variações dos gastos com alimentação e transporte.

Foi realizada uma comparação do IPVT com o TPI dos EUA indicando que, no Brasil, os preços elevaram-se de forma bem mais significativa. No período de 1998 a 2009, a taxa média de variação anual do IPVT foi de 9,83\% enquanto que a do TPI foi de 2,97\%. Analisando-se os preços relativos de viagens e turismo e a taxa de câmbio real entre Brasil e EUA, concluise que de 1998 a 2002, a desvalorização da taxa de câmbio real estimulou a entrada de turistas americanos. Posteriormente, em função da taxa de inflação doméstica e da forte valorização 
da taxa de câmbio nominal, observa-se uma forte valorização da taxa de câmbio real o que, explica até certo ponto, a redução da entrada de turistas americanos no Brasil.

Finalmente, deve-se observar que, em geral, os modelos de previsão de demanda de viagens e turismo utilizam o índice de preços ao consumidor no lugar de um índice de preços específico para viagens e turismo pois este tipo de índice não se encontra calculado para a maioria dos países. Embora possa parecer uma boa aproximação, o ideal é a utilização de um índice específico como é o caso do TPI e do IPVT.

\section{Referências}

ALLEN, R.G.D. Index Numbers in Theory and Practice. London. The Macmillan Press, 1975.

CARMO, H.C.E. Um Enfoque Integrado para Números-Índices Econômicos: Uma Aplicação ao Cálculo de Índices de Preços ao Consumidor no Município de São Paulo, no periodo de 1939-1986. Tese de Doutoramento IPE-USP (1988).

CARMO, H.C.E. Índice de Preços ao Consumidor: Teoria e Análise de Modelos Factíveis Considerando as Bases de Dados Disponíveis. Tese de Livre Docência Departamento de Economia da FEA-USP. 2004.

CROUCH, GEOFFREY I. Effect of Income and Price on International Tourism. Annals of Tourism Research, Vol. 19, pp. 643-664, 1992.University of Calgary, Canada.

DEATON, A MUELLBAUER, Economics and Consumer Behavior. Cambridge University Press, 1994.

DIEWERT, W. ERWIN. The Consumer Price Index and Index Number Purpose. Fifth Meeting of the International Working Group on Price Indices (The Ottawa Group), Reykjavik, Iceland, August 25-27, 1999.

DIEWERT, W. ERWIN. Index number. In: EATWELL, J. M., MILGATE, P. N. (eds.). The New Palgrave. Dictionary of Economics, New York: The MacMillian Press, v. 2, p. 767-780, 1988.

ENDO, SEITI KANEKO \& DO CARMO, HERON ESVAEL. Pesquisa de Orçamentos Familiares no Município de São Paulo. IPE. São Paulo, 1984.

IBGE. Série Relatórios Metodológicos. Vol.14. Sistema Nacional de índices de Preços ao Consumidor. Métodos de Cálculo. $5^{\text {a }}$ Ed. Rio de Janeiro, 2007.

IBGE. Série Relatórios Metodológicos. Vol.34. Sistema Nacional de índices de Preços ao Consumidor. Estruturas de Ponderação a Partir da Pesquisa de Orçamentos familiares 20022003. Rio de Janeiro, 2005. 
KEYNES, J.M. A Treatise on Money. In Royal Economic Society (1971 ed.) The Collected Writings of John Maynard Keynes. London: Cambridge University Press,1930 e. 1971.

KONÜS, A.A. The Problem of the True Index of the Cost of Living. Econometrica 7, 10$29,1924$.

LIM, CHRISTINE. Review of International Tourism Demand Models. Annals of Tourism Research, Vol. 24, No. 4, pp. 835-849, 1997. University of Western Australia, Australia.

ONG, CHRISTINE. Tourism Demand Models: a Critique. Mathematics and Computers in Simulation 39 (1995) 367-372. University of Western Australia. Department of Economics.

POLLAK, R. A. The Theory of Cost of Living Index. Oxford University Press , 1989

RABAHY, WILSON ABRAHÃO \& KADOTA, DÉCIO K. Caracterização $e$ Dimensionamento do Turismo Doméstico no Brasil. 2006. Metodologia e Desenvolvimento. FIPE-EMBRATUR. Brasília, setembro/2006.

SONG, HAIYAN \& LIB, GANG. Tourism Demand Modelling and Forecasting. A Review of Recent Research. Tourism Management 29 (2008) 203-220.

\section{Recebido 12/12/2010}

Aprovado 23/03/2011 